\title{
WNT antagonist, DKK2, is a Notch signaling target in intestinal stem cells: Augmentation of a negative regulation system for canonical WNT signaling pathway by the Notch-DKK2 signaling loop in primates
}

\author{
MASUKO KATOH ${ }^{1}$ and MASARU KATOH ${ }^{2}$ \\ ${ }^{1}$ M\&M Medical BioInformatics, Hongo 113-0033; ${ }^{2}$ Genetics and Cell Biology Section, \\ National Cancer Center Research Institute, Tokyo 104-0045, Japan \\ Received August 2, 2006; Accepted October 23, 2006
}

\begin{abstract}
Notch and WNT signaling pathways are key components of the stem cell signaling network. Canonical WNT signaling to intestinal progenitor cells leads to transcriptional activation of the JAGl gene, encoding Serratetype Notch ligand. JAG1 then binds to the Notch receptor on adjacent stem cells to induce Notch receptor proteolyses for the release of Notch intracellular domain (NICD). NICD is associated with CSL/RBPSUH and Mastermind (MAML1, MAML2, or MAML3) to activate Notch target genes, such as HES1 and HES5. Although WNT-dependent Notch signaling activation in intestinal stem cells is clarified, the effects of Notch signaling activation on WNT signaling in progenitor cells remain unclear. We searched for Notch-response element (NRE) in the promoter region of genes encoding secreted WNT signaling inhibitors, including $D K K 1, D K K 2, D K K 3, D K K 4$, SFRP1, SFRP2, SFRP3, SFRP4, SFRP5 and WIF1. Double NREs were identified within human $D K K 2$ promoter by bioinformatics and human intelligence (Humint). The human $D K K 2$ gene was characterized as Notch signaling target in intestinal stem cells. Because DKK2 is a key player in the stem cell signaling network, the $D K K 2$ gene at human chromosome $4 \mathrm{q} 25$ is a candidate tumor suppressor gene inactivated due to epigenetic silencing and/or deletion. The chimpanzee $D K K 2$ gene was identified within the NW_105990.1 genome sequence, while the cow $D k k 2$ gene was identified within the AC156664.2 and AC158038.2 genome sequences. Chimpanzee DKK2 and cow Dkk2 showed 98.5\% and 95.8\% total-aminoacid identity with human DKK2, respectively. Double NREs in human $D K K 2$ promoter were conserved in chimpanzee
\end{abstract}

Correspondence to: Dr Masaru Katoh, Genetics and Cell Biology Section, National Cancer Center Research Institute, 5-1-1 Tsukiji, Chuo-ku, Tokyo 104-0045, Japan

E-mail: mkatoh-kkr@umin.ac.jp

Key words: Notch, WNT, FGF, stem cell signaling network, integrome network, systems biology
$D K K 2$ promoter, partially in rat $D k k 2$ promoter, but not in cow and mouse $D k k 2$ promoters. The Notch-DKK2 signaling loop, created or potentiated in primates, was complementary to WNT-DKK1 and BMP-IHH-SFRP1 signaling loops for negative regulation of canonical WNT signaling pathway. Together, these facts indicate that $D K K 2$ promoter evolution resulted in the augmentation of a WNT negative regulation system in primates.

\section{Introduction}

Interaction among Notch (1-3), WNT (4-6), FGF (7-9), BMP (10-12) and Hedgehog (13-15) signaling pathways constitutes the stem cell signaling network. Canonical Notch signaling in intestinal stem cells leads to Notch receptor proteolyses for the release of Notch intracellular domain (NICD), which is associated with CSL/RBPSUH and Mastermind to activate Notch target genes, such as HES1 and HES5 $(16,17)$.

Canonical WNT signaling to intestinal progenitor cells leads to transcriptional activation of the $J A G l$ gene, encoding Serrate-type Notch ligand (18). JAG1 then binds to the Notch receptor on adjacent stem cells to induce Notch signaling activation. Because canonical WNT signaling is implicated in cell-fate determination, dysregulation of the canonical WNT signaling pathway leads to carcinogenesis. The WNT-DKK1 signaling loop (19) and BMP-IHH-SFRP1 signaling loop (20) are known as components of a negative regulation system for canonical WNT signaling pathway.

Although WNT-dependent Notch signaling activation in stem cells is clarified, the effects of Notch signaling activation in stem cells on WNT signaling progenitor cells remain unclear. Here, we searched for Notch-response element (NRE) in the promoter region of human genes encoding secreted WNT signaling inhibitors. Double NREs were identified within human $D K K 2$ promoter. Based on promoter and expression domain analyses, human $D K K 2$ was characterized as the target of Notch signaling pathway in intestinal stem cells. Chimpanzee $D K K 2$ and cow $D k k 2$ genes were then identified. Comparative genomics analyses revealed $D K K 2$ promoter evolution in primates. The Notch-DKK2 signaling loop, created or potentiated in primates, was complementary to WNT-DKK1 
A

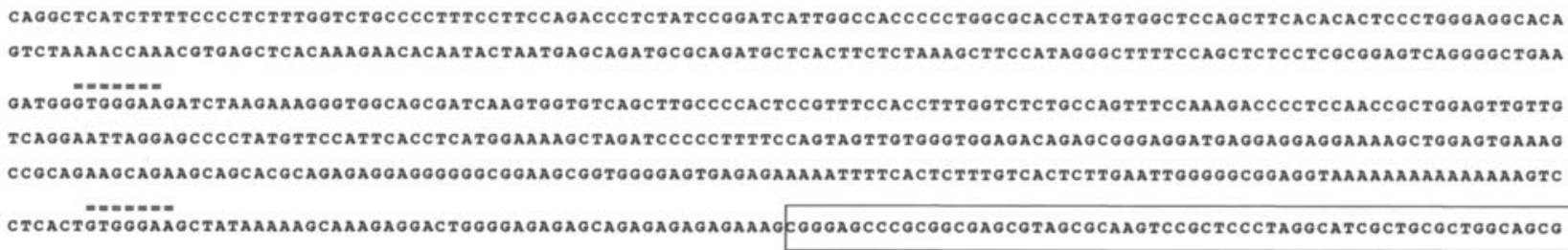

B

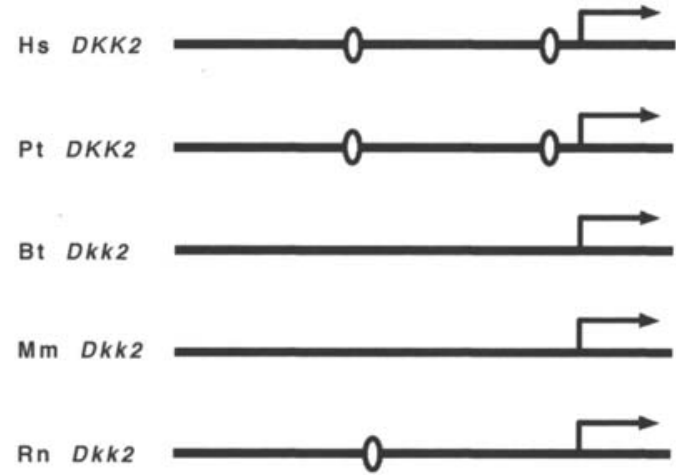

C

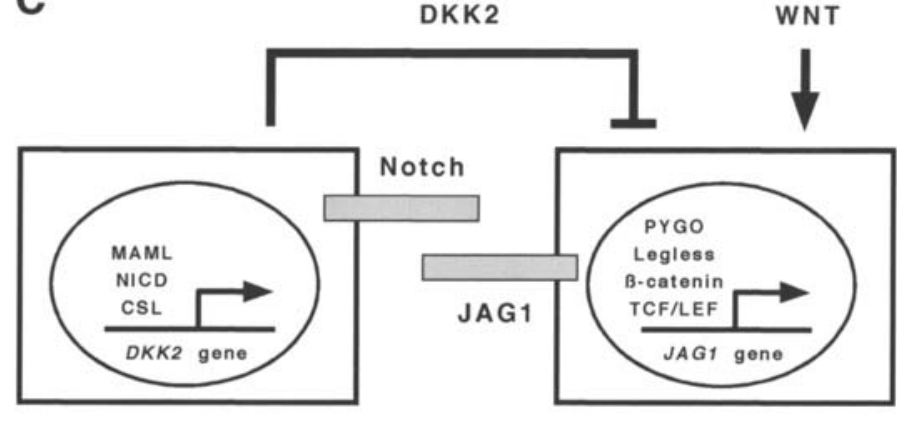

Stem cells

Progenitor cells

Figure 1. Promoters of mammalian DKK2 orthologs. (A) Human DKK2 promoter. Double Notch-response elements (NREs) are shown by double over-lines. Region corresponding to exon 1 of the human $D K K 2$ gene is boxed. (B) Schematic representation of promoters of mammalian DKK2 orthologs. Hs, human; Pt, chimpanzee; Bt, cow; Mm, mouse; Rn, rat. NRE is shown by open oval. (C) Notch-DKK2 signaling loop. Canonical WNT signaling in progenitor cells activates JAG1 expression for Notch signaling activation in stem cells. Notch signaling in stem cells then activates DKK2 expression for canonical WNT signaling downregulation in progenitor cells. The Notch-DKK2 signaling loop functions as a negative feedback loop for the canonical WNT signaling pathway.

and BMP-IHH-SFRP1 signaling loops for negative regulation of the canonical WNT signaling pathway.

\section{Materials and methods}

Promoter regions of genes encoding secreted WNT signaling inhibitors. DKK1,DKK2, DKK3, DKK4, SFRP1, SFRP2, SFRP3, SFRP4, SFRP5 and WIF1 genes encode secreted-type WNT signaling inhibitors. Promoter regions of human $D K K 1$, DKK2, DKK3, DKK4, SFRP1, SFRP2, SFRP3, SFRP4, SFRP5 and WIF1 genes are located within the AC009986.10, AP001819.2, AC124276.5, AF170802.6, AC016868.8, AC020703.7, AC108514.3, AC018634.3, AL358938.8, and AC026124.35 genome sequences, respectively, as previously described (20).

Screening for Notch target genes. NRE within the 5'-flanking promoter region was searched for based on bioinformatics and human intelligence (Humint) as described previously for the search of canonical WNT target genes (21-23).

Identification of chimpanzee and cow DKK2 orthologs. Chimpanzee and cow genome sequences homologous to human DKK2 were searched for with the BLAST programs as previously described (24-26). Exon-intron boundaries were determined by examining the consensus sequence of exonintron junctions ("gt ..... ag" rule of intronic sequence) and the codon usage within the coding region as previously described (27-29). Coding sequences of chimpanzee DKK2 and cow Dkk2 were determined by assembling exonic regions.

Comparative integromics analyses. Promoters and expression domains of mammalian DKK2 orthologs were compared as previously described $(20,30)$.

\section{Results}

Screening of NRE within promoter regions of genes encoding secreted WNT signaling inhibitors. Among 5'-promoter regions of DKK1,DKK2,DKK3,DKK4,SFRP1,SFRP2, SFRP3, SFRP4, SFRP5 and WIF 1 genes, double NREs were identified within the promoter region of the human $D K K 2$ gene (Fig. 1A). NREs within $D K K 2$ promoter were located approximately 400 and 50 bp upstream of the transcriptional start site. Human $D K K 2$ was characterized as the Notch target gene.

Comparative genomics on DKK2 orthologs. We previously reported the identification and characterization of the rat $D k k 2$ gene in silico (29). Other mammalian $D K K 2$ othologs were further searched for in this study. The chimpanzee $D K K 2$ gene was identified within the NW_105990.1 genome sequence, while the cow Dkk2 gene was identified within AC156664.2 and AC158038.2 genome sequences. Exon-intron boundaries of chimpanzee $D K K 2$ and cow $D k k 2$ genes were determined based on the consensus sequence of exon-intron junctions. Both the chimpanzee DKK2 gene (Fig. 2A) and cow Dkk2 gene (Fig. 2B) were found to consist of four exons. The complete coding sequence (CDS) of chimpanzee DKK2 (Fig. 2C) and that of cow Dkk2 (Fig. 2D) were determined by assembling nucleotide sequences of four exons. The chimpanzee $D K K 2$ gene as well as cow $D k k 2$ gene were found to encode 259-amino-acid protein. Chimpanzee DKK2 and cow Dkk2 showed $98.5 \%$ and $95.8 \%$ total-amino-acid identity with human DKK2, respectively.

NRE within the promoter region of mammalian DKK2 orthologs was next searched for. Double NREs were identified within chimpanzee $D K K 2$ promoter, which were approximately 400 and $50 \mathrm{bp}$ upstream of the putative transcriptional start site. Single NRE was identified within rat $D k k 2$ promoter, 
A

\begin{tabular}{|c|c|c|}
\hline $\begin{array}{c}\text { Exon } \\
\text { No. }\end{array}$ & $\begin{array}{l}\text { Nucleotide sequence of chimpanzee DKK2 } \\
\text { gene around exon - intron boundarles }\end{array}$ & $\begin{array}{l}\text { Nucleotide position of exon } \\
\text { in genome sequence }\end{array}$ \\
\hline $\begin{array}{l}1 \\
2 \\
3 \\
4\end{array}$ & 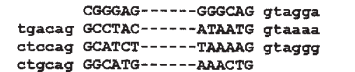 & $\begin{array}{l}11321317-11320391 \text { of } \mathrm{NW}-105990.1 \\
1121521-11215571 \text { of } \mathrm{NW}-105900.1 \\
11214472-11214317 \text { of } \mathrm{NW}-105990.1 \\
11213981-11211581 \text { of } \mathrm{NW} 105990.1\end{array}$ \\
\hline
\end{tabular}

B

\begin{tabular}{|c|c|c|}
\hline $\begin{array}{l}\text { Exon } \\
\text { No. }\end{array}$ & $\begin{array}{l}\text { Nucleotide sequence of cow Dkk2 } \\
\text { gene around exon - intron boundarles }\end{array}$ & $\begin{array}{l}\text { Nucleotide positlon of exon } \\
\text { in genome sequence }\end{array}$ \\
\hline $\begin{array}{l}1 \\
2 \\
3 \\
4\end{array}$ & 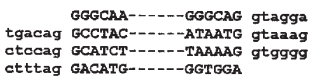 & $\begin{array}{r}176464=177031 \text { of AC156664.2 } \\
99617=99767 \text { of AC158038.2 } \\
101072=101227 \text { of AC158038.2 } \\
103780-104099 \text { of AC158038.2 }\end{array}$ \\
\hline
\end{tabular}

C

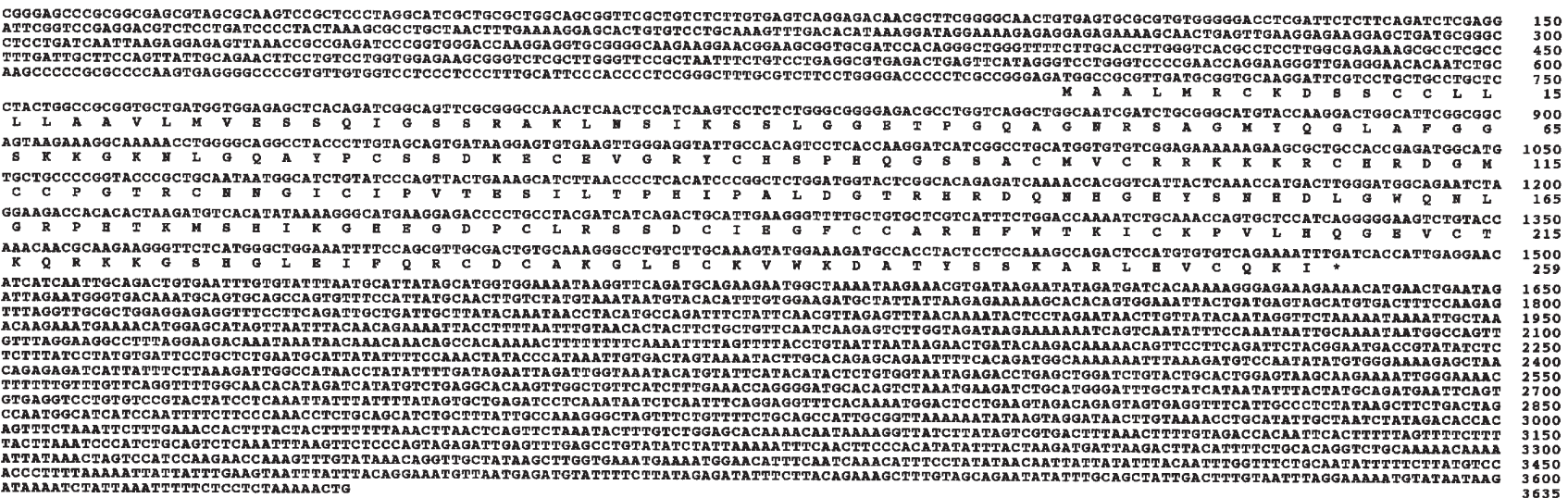

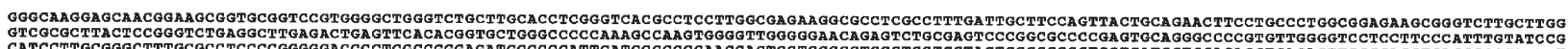

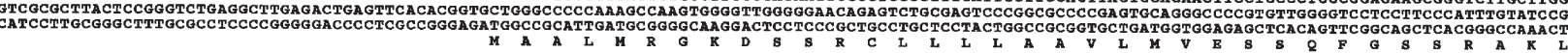

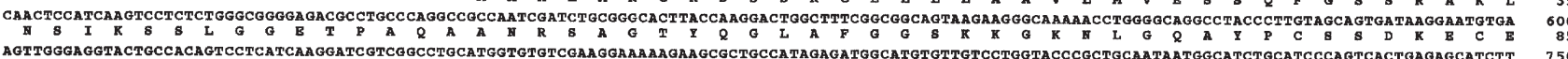

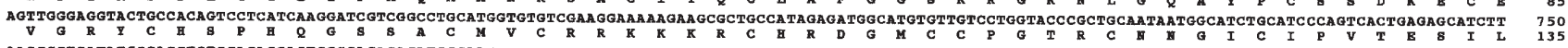

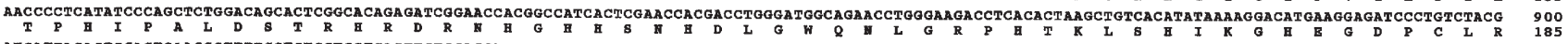

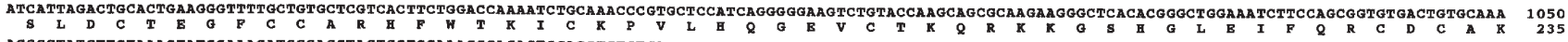

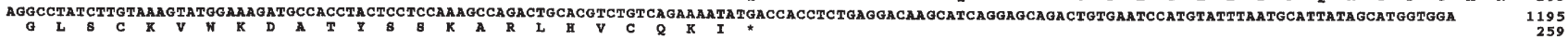

Figure 2. Chimpanzee $D K K 2$ and cow $D k k 2$ genes. (A) Exon-intron structure of the chimpanzee $D K K 2$ gene. (B) Nucleotide and amino-acid sequences of chimpanzee DKK2 complete CDS. (C) Exon-intron structure of the cow Dkk2 gene. (D) Nucleotide and amino-acid sequences of cow Dkk2 complete CDS
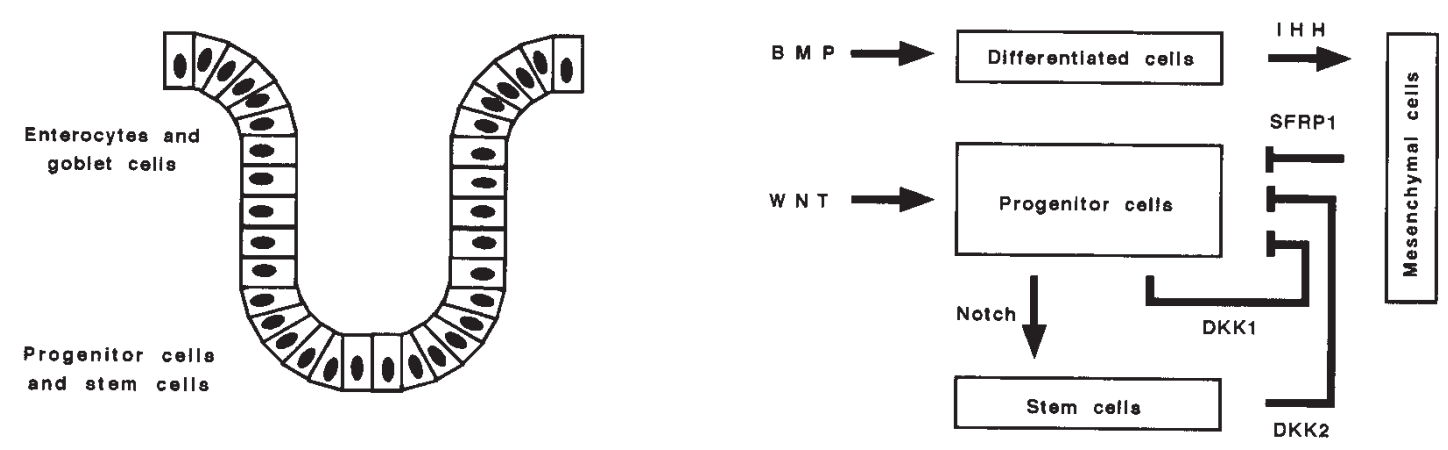

Figure 3. Multi-layer regulation system for the canonical WNT signaling pathway in human intestine. (Left) Location of stem, progenitor, and differentiated cells in the human intestine. (Right) Diagram of WNT signaling regulation system. Notch-DKK2, WNT-DKK1, and BMP-IHH-SFRP1 signaling loops constitute the multi-layer regulation system for the canonical WNT signaling pathway in human intestine.

which was approximately 400 bp upstream of the putative transcriptional start site. NRE was not identified within cow and mouse $D k k 2$ promoters. Double NREs within human $D K K 2$ promoter were conserved in chimpanzee $D K K 2$ promoter, partially in rat $D k k 2$ promoter, but not in cow and mouse $D k k 2$ promoters (Fig. 1B). Primate $D K K 2$ orthologs were characterized as the target of Notch signaling pathway.

Integromics analyses on human DKK2 and mouse Dkk2. Notch signals are activated in the intestinal stem cell region, and double NREs were identified within human $D K K 2$ promoter, but not within mouse promoter, as mentioned above. Byun et al reported that human $D K K 2$ is expressed in colonic stem cell region (31). Gregorieff et al reported that mouse $D k k 2$ is not expressed in normal colonic mucosa (32). Together, these facts indicate that human $D K K 2$, but not mouse $D k k 2$, was the Notch target gene in intestinal stem cells (Fig. 1C).

Multi-layer system for canonical WNT signaling inhibition. In addition to WNT-DKK1 and BMP-IHH-SFRP1 signaling loops $(19,20)$, Notch-DKK2 signaling loops were identified as the novel mechanism for canonical WNT signaling inhibition in primates (Fig. 1C). These facts indicate that the negative regulation system for the canonical WNT signaling pathway consists of multiple signaling loops, such as Notch-DKK2, WNT-DKK1, and BMP-IHH-SFRP1 (Fig. 3).

\section{Discussion}

NRE in the promoter region of human genes encoding secreted WNT signaling inhibitors was searched for in this study, 
because the effects of Notch signaling activation in intestinal stem cells on WNT signaling in intestinal progenitor cells remain unclear. Among 10 human genes encoding secreted WNT signaling inhibitor, double NREs were identified within human DKK2 promoter (Fig. 1A). On the other hand, NRE was not identified within mouse $D k k 2$ promoter (Fig. 1B). Human DKK2 is expressed in colonic stem cell region (31), while mouse $D k k 2$ is not expressed in normal colonic mucosa (32). Although mouse Dkk2 was not the target gene of Notch signaling pathway, human $D K K 2$ was characterized as the Notch target gene, especially in the intestinal stem cells (Fig. 1C).

Secreted WNT signaling inhibitors are implicated in the regulation of WNT signaling pathway, which plays key roles in a variety of processes, such as embryogenesis, maintenance of adult tissue homeostasis, tissue repair during chronic persistent inflammation, and carcinogenesis (4-6,33-35). Because aberrant WNT signaling activation leads to carcinogenesis, SFRPI, $D K K 1$ and WIF1 genes, encoding secreted WNT signaling inhibitors, are claimed as tumor suppressor genes inactivated due to $\mathrm{CpG}$ hypermethylation. The $D K K 2$ gene is located at the human chromosome $4 \mathrm{q} 25$ region, which is deleted in colorectal cancer, breast cancer, lung cancer, and cervical cancer (36-39). Epigenetic changes occur at the early stage during multi-stage carcinogenesis associated with chronic persistent inflammation and/or aging, and then genetic alterations occur to augment the malignant potential of cancer cells $(40,41)$. Therefore, $D K K 2$ is a candidate tumor suppressor gene inactivated due to epigenetic silencing and/or deletion.

We characterized rat $D k k 2$ (29), chimpanzee $D K K 2$ (Fig. 2B), and cow Dkk2 (Fig. 2D). Krupnik et al characterized human $D K K 2$ (42). Monaghan et al characterized mouse $D k k 2$ (43). Human DKK2 showed 98.5\%, 95.8\%, 95.8 and 95.4\% total-amino-acid identity with chimpanzee DKK2, cow Dkk2, rat Dkk2 and mouse Dkk2, respectively. Amino-acid sequences of DKK2 orthologs were well-conserved among mammals.

Double NREs in human $D K K 2$ promoter were conserved in chimpanzee $D K K 2$ promoter, partially in rat $D k k 2$ promoter, but not in cow and mouse $D k k 2$ promoters (Fig. 1B). Comparative genomics analyses on the promoter region of $D K K 2$ orthologs revealed that the Notch-DKK2 signaling loop was created or potentiated in primates. The Notch-DKK2 signaling loop identified in this study was complementary to WNTDKK1 and BMP-IHH-SFRP1 signaling loops for negative regulation of the canonical WNT signaling pathway (Fig. 3). Together, these facts indicate that $D K K 2$ promoter evolution resulted in the augmentation of a WNT negative regulation system in primates.

\section{References}

1. Artavanis-Tsakonas S, Rand MD and Lake RJ: Notch signaling: Cell fate control and signal integration in development. Science 284: 770-776, 1999.

2. Radtke F and Raj K: The role of Notch in tumorigenesis: Oncogene or tumor suppressor? Nat Rev Cancer 3: 765-767, 2003.

3. Li JL and Harris AL: Notch signaling from tumor cells: a new mechanism of angiogenesis. Cancer Cell 8: 1-3, 2005.

4. Katoh M: Regulation of WNT signaling molecules by retinoic acid during neuronal differentiation in NT2 cells: Threshold model of WNT action. Int J Mol Med 10: 683-687, 2002.

5. Katoh M: WNT2B: Comparative integromics and clinical application. Int J Mol Med 16: 1103-1108, 2005.
6. Katoh M: WNT/PCP signaling pathway and human cancer. Oncol Rep 14: 1583-1588, 2005.

7. Katoh M: WNT and FGF gene clusters. Int J Oncol 21: 1269-1273, 2002.

8. Katoh M and Katoh M: FGF signaling network in the gastrointestinal tract. Int J Oncol 29: 163-168, 2006.

9. Katoh M and Katoh M: Cross-talk of WNT and FGF signaling pathways at GSK3ß to regulate $\beta$-catenin and SNAIL signaling cascades. Cancer Biol Ther 5: 1059-1064, 2006.

10. Katoh M and Terada M: Overexpression of bone morphogenetic protein (BMP)-4 in gastric cancer cell lines of poorly differentiated type. J Gastroenterol 31: 137-139, 1996.

11. Katoh Y and Katoh M: Comparative genomics on BMP4 orthologs. Int J Oncol 27: 581-585, 2005.

12. Katoh Y and Katoh M: Comparative integromics on BMP/GDF family. Int J Mol Med 17: 951-955, 2006.

13. Pasca di Magliano $M$ and Hebrok M: Hedgehog signalling in cancer formation and maintenance. Nat Rev Cancer 3: 903-911, 2003.

14. Bijlsma MF, Spek CA and Peppelenbosch MP: Hedgehog: an unusual signal transducer. Bioessays 26: 387-394, 2004.

15. Katoh $\mathrm{Y}$ and Katoh M: Hedgehog signaling in gastric cancer. Cancer Biol Ther 4: 1050-1054, 2005.

16. Feder JN, Li L, Jan LY, et al: Genomic cloning and chromosomal localization of $H R Y$, the human homolog to the Drosophila segmentation gene, hairy. Genomics 20: 56-61, 1994.

17. Katoh $\mathrm{M}$ and Katoh M: Identification and characterization of human HES2, HES3, and HES5 genes in silico. Int J Oncol 25: 529-534, 2004.

18. Katoh M and Katoh M: Notch ligand, JAG1, is evolutionarily conserved target of canonical WNT signaling pathway in progenitor cells. Int J Mol Med 17: 681-685, 2006.

19. Katoh $\mathrm{Y}$ and Katoh M: Comparative genomics on DKK1 orthologs. Int J Oncol 27: 275-279, 2005.

20. Katoh Y and Katoh M: WNT antagonist, SFRP1, is Hedgehog signaling target. Int J Mol Med 17: 171-175, 2006.

21. Katoh M and Katoh M: Comparative genomics on FGF8, $F G F 17$, and FGF18 orthologs. Int J Mol Med 16: 493-496, 2005.

22. Katoh $\mathrm{M}$ and Katoh M: Comparative genomics on FGF2O orthologs. Oncol Rep 14: 287-290, 2005.

23. Katoh Y and Katoh M: FGF signaling inhibitor, SPRY4, is evolutionarily conserved target of WNT signaling pathway in progenitor cells. Int J Mol Med 17: 529-532, 2006.

24. Katoh M: Paradigm-shift in gene-finding method: From benchtop approach to desk-top approach. Int J Mol Med 10: 677-682, 2002.

25. Katoh $\mathrm{M}$ and Katoh M: Identification and characterization of human PRICKLE1 and PRICKLE2 genes as well as mouse Prickle1 and Prickle2 genes homologous to Drosophila tissue polarity gene prickle. Int J Mol Med 11: 249-256, 2003.

26. Katoh $M$ and Katoh $M$ : Identification and characterization of human BCL9L gene and mouse Bcl9l gene in silico. Int $\mathrm{J}$ Mol Med 12: 643-649, 2003.

27. Katoh $M$ and Katoh $M$ : Identification and characterization of human HESL, rat Hesl and rainbow trout hesl genes in silico. Int J Mol Med 14: 747-751, 2004

28. Katoh Y and Katoh M: Comparative genomics on SFRP1 orthologs. Int J Oncol 27: 861-865, 2005.

29. Katoh Y and Katoh M: Comparative genomics on $D K K 2$ and DKK4 orthologs. Int J Mol Med 16: 477-481, 2005.

30. Katoh Y and Katoh M: Comparative genomics on HHIP family orthologs. Int J Mol Med 17: 391-395, 2006.

31. Byun T, Karimi M, Marsh JL, et al: Expression of secreted Wnt antagonists in gastrointestinal tissues: potential role in stem cell homeostasis. J Clin Pathol 58: 515-519, 2005.

32. Gregorieff A, Pinto D, Begthel H, et al: Expression pattern of WNT signaling components in the adult intestine. Gastroenterology 129: 626-638, 2005.

33. Heller RS, Klein T, Ling Z, Heimberg H, Katoh M, Madsen OD and Serup P: Expression of WNT, Frizzled, sFRP, and DKK genes in adult human pancreas. Gene Expr 11: 141-147, 2003.

34. Garciadiego-Cazares D, Rosales C, Katoh M and ChimalMonroy J: Coordination of chondrocyte differentiation and joint formation by $\alpha 5 \beta 1$ integrin in the developing appendicular skeleton. Development 131: 4735-4742, 2004.

35. Swain RK, Katoh M, Medina A and Steinbeisser H: Xenopus frizzled-4S, a splicing variant of Xfz4, is a context-dependent activator and inhibitor of Wnt/B-catenin signaling. Cell Commun Signal 3: 12, 2005. 
36. Shivapurkar N, Maitra A, Milchgrub S, et al: Deletions of chromosome 4 occur early during the pathogenesis of colorectal carcinoma. Hum Pathol 32: 169-177, 2001.

37. Shivapurkar N, Sood S, Wistuba II, et al: Multiple regions of chromosome 4 demonstrating allelic losses in breast carcinomas. Cancer Res 59: 3576-3580, 1999.

38. Park SY, Kim YH, In KH, et al: Chromosomal aberrations in Korean nonsmall cell lung carcinomas: degenerate oligonucleotide primed polymerase chain reaction comparative genomic hybridization studies. Cancer Genet Cytogenet 152: 153-157, 2004.

39. Costa C, Fuste P, Alameda F, et al: Study of chromosomal abnormalities in 11 cases of cervical dysplasia using comparative genomic hybridization on cotton-lint cervical samples. Cancer Genet Cytogenet 164: 61-65, 2006.
40. Baylin SB and Ohm JE: Epigenetic gene silencing in cancer - a mechanism for early oncogenic pathway addiction? Nat Rev Cancer 6: 107-116, 2006.

41. Katoh M: Bioinformatics for cancer management in the postgenome era. Technol Cancer Res Treat 5: 169-176, 2006.

42. Krupnik VE, Sharp JD, Jiang C, et al: Functional and structural diversity of the human Dickkopf gene family. Gene 238: 301-313, 1999.

43. Monaghan AP, Kioschis $\mathrm{P}, \mathrm{Wu} \mathrm{W}$, et al: Dickkopf genes are co-ordinately expressed in mesodermal lineages. Mech Dev 87 : 45-56, 1999. 